\title{
Personalidad y educación: caso estudiantes de pedagogía
}

\section{Personality and Education: case of pedagogy students}

\author{
Evodio Juber Orozco-Edelman ${ }^{1}$
}

\begin{abstract}
Resumen
El estudio respondió a la pregunta ¿Cuáles son los rasgos de personalidad de los estudiantes participantes? Para el efecto se trabajó el objetivo de determinar los rasgos de personalidad de estudiantes de Pedagogía, de la Facultad de Humanidades, de la Universidad de San Carlos de Guatemala. Se administró la prueba: "Unidades de la personalidad desde la emoción y la actitud UPEA", a 62 estudiantes tanto de la jornada vespertina y plan sabatino. Los resultados globales de la prueba evidenciaron que la mayoría de los estudiantes, se inclinan al análisis y la reflexión, predominó el tipo de personalidad abstractiva.
\end{abstract}

\section{Palabras clave}

Actitudes, emociones, pruebas psicológicas, personalidad.

\begin{abstract}
The study answered the question, what are the personality traits of the participating students? For the effect it worked in the objective of determining the personality traces of Pedagogy students, from the Humanities Faculty, of the University of San Carlos of Guatemala. The test applied was named: "Units of the personality from the emotion and attitude UPEA", to 62 students of both the afternoon session and the Saturday plan. The overall results of the test showed that the majority of students, inclined to analysis and reflection, predominated the type of abstractive personality.
\end{abstract}

\section{Keywords}

Attitudes, emotions, psychological tests, personality.

1 Evodio Juber Orozco-Edelman. Profesor Titular del Departamento de Investigación Educativa de la División de Desarrollo Académico, Universidad de San Carlos de Guatemala. Psicólogo con maestría en Antropología Social y Doctor en Educación. Correo electrónico: evojuble@gmail.com

ID: http://orcid.org/0000-0002-1268-509X 
RECIE. Revista Electrónica Científica de Investigación Educativa Vol. 4, núm. 2, enero-diciembre 2019, pp. 1073-1081.

\section{Introducción}

La educación para desarrollarse y fortalecerse necesita de un esfuerzo intencionado, de un acto de voluntad socio-cultural. Estos aspectos tienen relación con los tres componentes del proceso de educativo: el heurístico que tiene que ver con la investigación de la verdad, el didáctico, que se relaciona con promover y facilitar el aprendizaje del conocimiento, y el poyiético, que consiste en aplicar el conocimiento a la realidad y a la acción social (González, 2001, González, 2007). Se observa en el contexto sociocultual, que existe ausencia de vínculos de los aspectos de la personalidad con diferentes campos de convivencia de las relaciones humanas, en particular con la educación.

Existe la neurociencia de la personalidad, está es un área de investigación de origen relativamente reciente. La tesis nuclear de esta disciplina sostiene que cada factor de la personalidad tiene un componente cerebral estructural y funcional (Restrepo, 2015). Asimismo, existe el modelo de los cinco factores que permite explorar de manera más completa esa relación entre bienestar subjetivo y personalidad, desde el afecto positivo y negativo y satisfacción con la vida (Moran, Fínez y Fernández, 2017) Asimismo esta la postura que entre la influencia mutua de ciertos rasgos de personalidad, se consentirían miradas diferenciales a las dimensiones temporales de la vida vivida y por vivir, porque el perfil de perspectiva temporal contribuye a la identidad con mayor o menor grado de adaptación e integralidad, aunado a que la tipología de la personalidad afectiva es apropiada para el conocimiento del bienestar subjetivo de los estudiantes universitarios (Vásquez, Difabio y Noriega, 2016; Moran, Fínez y Fernández, 2017).

Además las emociones, sentimientos y afectos, como parte de la personalidad inciden en la educación. Según Caicedo "uno de los aportes que quizá puede contribuir más en la educación es el reconocimiento confirmado del papel que juegan las emociones en el aprendizaje y el significado" (2012 p. 149). Respecto de las emociones, Tirapu (2008) manifiesta que la emoción es un conjunto de habilidades psicológicas que influyen en nuestro comportamiento, como el autocontrol, el estado de ánimo, el entusiasmo, la auto-motivación, la empatía, la autoconciencia, la perseverancia, la capacidad para interaccionar con los demás. En tal sentido, como un primer momento es fundamental responder a la pregunta ¿Cuáles son los rasgos de personalidad de los estudiantes de pedagogía participantes? 


\section{Personalidad: emoción y actitud}

Existen aspectos teóricos que fundamentan la estructura de la personalidad. Son fundamentales las teorías de Jung (2000) y la Marston (1928) El primero a partir de la actitud y el segundo desde la emoción. Existen dos aspectos actitudinales, el introvertido y el extravertido, la peculiaridad se debe a que la persona se orienta principalmente por la función que en él o ella se halla más diferenciada. Estos son los tipos generales de actitud, se distinguen por la dirección de su interés, por el movimiento de la libido (Jung, 2000). Las citas que se presentan de William Marston, son una traducción libre con el apoyo de Enrique Gordillo, del libro "Emotion of normal people", publicado en 1928. Al respecto esta autor planteó que hay dos principios integradores, que parecen operar independientemente y que pueden combinarse uno con el otro. Estos principios son la Alianza y Antagonismo del Estímulo externo hacia el Yo. El otro principio tiene que ver con la intensidad. Un volumen inferior de la intensidad del Estímulo, evoca aumento en el volumen del Yo; y la intensidad superior del Estímulo, evoca decrecimiento del volumen del Yo (Marston citado por OrozcoEdelman y González-Oliva, 2016).

Jung (2000), profundiza en los dos componentes de la personalidad al plantear que el comportamiento del introvertido frente al objeto es abstractivo, en el fondo tiende siempre a sustraer al objeto la libido, como si temiese una prepotencia del objeto. Por su parte el extravertido tiene un comportamiento positivo con el objeto. Afirma la importancia del objeto, en la medida en que continuamente orienta su actitud subjetiva hacia el objeto y la refiere a él. En el fondo el valor poseído por el objeto no es nunca suficiente para el extravertido y por ello es preciso elevar ese valor. Ambos tipos son tan distintos.

Asimismo, Jung (2000), manifestó que Introvertido y Extrovertido forman parte de la naturaleza humana, todo el mundo conoce personalidades cerradas, impenetrables, tímidas a menudo, que contrastan con los caracteres abiertos, sociables, a menudo alegres o al menos amables y accesibles, que se llevan bien con todos o que se pelean con todos, influyen y se dejan influir. Unas veces resulta más clara y otra más confusa identificar estas tipologías. Esta clase de naturaleza humana se encuentra no sólo entre los cultos, sino en general en todas las capas de la población. Tampoco la diferencia de los sexos modifica este hecho, no es un asunto de la conciencia, sino que los tipos se reparten de un modo aparentemente indiscriminado. En una familia uno de los hijos es introvertido y el otro es extravertido, por eso, el tipo de actitud en cuanto fenómeno general y repartido de manera 
RECIE. Revista Electrónica Científica de Investigación Educativa Vol. 4, núm. 2, enero-diciembre 2019, pp. 1073-1081.

aparentemente casual, se debe su existencia, sin duda, a una causa inconsciente e instintiva.

Las Emociones Primarias son puntos nodales de series de emoción, donde las relaciones de Alianza y Antagonismo, y aumento o disminución del Yo, alcanzan el máximo, y comienzan a cambiar hacia el tipo opuesto de relación. Las Emociones Primarias generan condiciones que tienen que ver con el Cumplimiento, Dominio, Inducción y Sumisión. En el Cumplimiento, el Estímulo, su intensidad es antagonista y superior al Yo, esto genera desagrado. La respuesta del Yo disminuye de intensidad, y la compulsión antagónica del Yo que producía indiferencia, cambia a procesos de agradabilidad, en proporción al volumen e inter-fascinaciones que el Estímulo Superior cedió (Marston citado por Orozco-Edelman y GonzálezOliva, 2016).

En el Dominio, el Estímulo, su intensidad es antagonista e inferior al Yo, inicialmente desagradable. La respuesta del Yo, aumenta la intensidad, y la compulsión antagónica del Estímulo, esto produce amabilidad en proporción al éxito, coexistiendo con incomodidad. En la Inducción, el Estímulo, su intensidad es aliada e inferior al yo, (agradable). La respuesta del Yo va en aumento de intensidad y la compulsión aliada al Estímulo, cada vez más agradable. En la Sumisión, el Estímulo, su intensidad es aliada y superior al Yo (agradable). La respuesta del Yo disminuye de intensidad, y la compulsión aliada al Yo, cada vez es más agradable (Marston citado por Orozco-Edelman y González-Oliva, 2016).

Entre los tipos psicológicos hay cuatro funciones básicas como manifestación de la libido o energía, el pensamiento, sentimiento, sensación e intuición. De estas cuatro funciones surgen los tipos psicológicos de Intelectual, Sentimental, Sensorial e Intuitivo. Estos elaboran procesos del Si-Mismo, concepto empírico que hace referencia al conjunto íntegro de todos los fenómenos psíquicos que se dan en el ser humano. Expresa la unidad y totalidad de la personalidad global, a consecuencia de su componente inconsciente, y no puede ser consciente sino de manera parcial, por lo que el concepto de Sí-Mismo es empírico solo en parte. Este concepto engloba cosas experimentales y no experimentales o que aún no han sido experimentadas (Jung, 2000).

Jung (2000) y Marston (1928) fueron medulares para elaborar la prueba "Unidades de la personalidad desde la emoción y la actitud UPEA". Esta prueba a partir de las direccionalidades y/o tendencias, mide a partir de 4 cuadrantes la personalidad. El cuadrante I, se codifica como PgET.I, mide la extraversión y la preferencia de la persona por interactuar no 
personas sino con las tareas, buscano resultados. El cuadrante II, se codifica como PgER.II, mide también la tendencia a la extraversión y la preferencia de la persona por las relaciones interpersonales. El cuadrante III, se codifica como PgAT.III, mide la tendencia a la abstracción y las preferencias por las tareas mesuradas. El cuadrante IV, se codifica como PgAR.IV, mide también la tendencia a la abstracción y las preferencias por las relaciones tambien mesuradas (Jung, 2000, Marston, 1928; PDA, 2005, PDA, s.f; OrozcoEdelman et al., 2017).

\section{Participantes}

La muestra fue intencional, se hizo paso de aulas y se les invitó a los estudiantes de pedagogía del técnico (Profesorado de Enseñanza Media PEM) y de licenciatura, de la Facultad de Humanidades, de la Universidad de San Carlos de Guatemala, a participar en el proceso. Se administró la encuesta socio-demográfica a 142 estudiantes y por diversas causas algunos fueron abandonando el proceso. Los resultados que se presentan corresponden a 62, de los que 53 estudiantes (85\%) corresponden al sexo femenino y 9 (15\%) al masculino, con edades comprendidas entre los 20 y 45 años. El promedio de edad de los participantes es de 27.47, con una desviación estándar de 7.01. Aunque la edad máxima es de 45 años, la media y la mediana indican que es una población joven de 26 y 27 años de edad.

Respecto a la jornada 31 estudiantes eran de la jornada vespertina y plan sabatino (50\%). Con relación al estado civil de la población, la mayoría de los estudiantes se encuentran solteros (63\%). Situación que les beneficia para continuar y finalizar los estudios universitarios. Sin embargo, un grupo considerable de la población restante está casada (35\%). La mayor parte de este último grupo decidió estudiar Pedagogía en el plan sabatino, pues en muchos casos también trabaja. Del grupo de personas casadas, algunos indicaron no tener descendientes. Por el contrario, varios estudiantes solteros/as indicaron tener hijos.

La población que tiene descendencia señaló tener más compromisos sociales debido a sus hijos e hijas. La mayoría de esta población estudia en la jornada del plan sabatino. Estos datos evidencian que para algunos estudiantes, continuar su formación académica en la carrera de Pedagogía exige más sacrificios, y que la oportunidad de estudiar en el plan sabatino es muy significativa para su formación profesional y el mejoramiento de su condición socioeconómica. La mayoría de los 62 estudiantes que conforman la población tienen el título de magisterio (71\%), por lo que existe una relación estrecha entre la carrera de diversificado y sus estudios universitarios de pedagogía. Respecto del tipo de establecimiento, un mayor 
RECIE. Revista Electrónica Científica de Investigación Educativa Vol. 4, núm. 2, enero-diciembre 2019, pp. 1073-1081.

número de estudiantes de la muestra provienen de centros educativos privados. De igual manera la mayoría circunscribe su labor educativa a los centros educativos privados.

\section{Instrumentos}

Prueba "Unidades de la personalidad desde la emoción y la actitud UPEA"

Mide cuatro dimensiones o cuadrantes de la personalidad, consiste de 96 reactivos o ítems, tipo Likert de respuesta abierta, en una escala de 1, 4, 7 y 10. Para el análisis de confiabilidad se empleó el coeficiente de alpha de Cronbach, el coeficiente obtenido para la primera dimensión fue de .767 con una $\mathrm{p}<.005$, lo que indica que los ítems de ese constructo están altamente correlacionados. Para la segunda dimensión, se obtuvo un alpha de .775 con una $\mathrm{p}<.005$, altamente correlacionados; para la tercera dimensión, se obtuvo un coeficiente de .621, lo que indica que es cuestionable la consistencia de ese constructo; y por último se obtuvo una coeficiente de .723 para la cuarta dimensión, que indica que están altamente correlacionados (OrozcoEdelman y González-Oliva, 2016).

\section{Interpretaciones de los rasgos de personalidad estudiantil}

En el planteamiento de González (2002), Garbarini (2011), y Mitjáns (2014), se afirma que la personalidad tiene sus inicios en los procesos íntimos de auto-confianza y de relación con el contexto, desde la diferenciación, ya que por medio de ella se elaboran símbolos, signos, sentidos y representaciones valorizadas de la significatividad de la autoimagen, esto hace que el autoconcepto se consolide en el ser humano como un sistema auto-organizado y auto-regulado de las configuraciones subjetivas a nivel psíquico.

Según los principios integradores de la personalidad, hay personas extrovertidas e introvertidas y/o proactivas y reactivas. En los resultados de la prueba UPEA, En el cuadrante PgET.I, de los 62 estudiantes, 13 (21\%) se ubicó en esta dimensión, son estudiantes extrovertidos y buscan la acción, ven la vida para alcanzar resultados, siempre están pensando en nuevas tareas, la extroversión las hace valerosas, audaces y no vacilan en tomar decisiones que valgan la pena, no les atemoriza la adversidad, más bien las estimula y su tenacidad les ayuda a triunfar. Son personas que tienen bastante confianza en sí mismas, lo que la hace personas fuertes, trabajadoras, exitosas, aventureras y valientes.

En el cuadrante PgER.II, se ubicaron 10 estudiantes (17\%), son también extrovertidos que buscan la acción, sin embargo sus preferencias las dirigen hacia las relaciones interpersonales, por eso son personas 
calurosas, alegres, seductoras y espontáneas, con un auténtico agrado por la gente, se fijan más en lo bueno de las personas, las cosas y el aspecto positivo de la vida, esto las hace muy optimistas y entusiastas. Por eso se les percibe como encantadoras, agradables, atentas, expresivas y amistosas. Les gusta propiciar que la gente se sienta bien, se mantienen de buen humor y no tienen restricciones en expresar sus sentimientos y pensamientos. Buscan la diversión y el contacto con otras personas a quienes puede entretener y entusiasmar (Marston, 1928; Jung, 2000; PDA, 2005; PDA, s.f; Bradberry, 2008; Orozco-Edelman et al., 2017).

En el cuadrante PgAT.III, se ubican 18 estudiantes (30\%), son abstractivos/introvertidos, esto propicia que sean reflexivas y busquen la estabilidad, por eso son personas tranquilas, invariables, serenas y persistentes para terminar las tareas. No les gustan los cambios de ninguna clase y prefieren escuchar y hacer. Son muy confiables, leales, apacibles, tácticas y organizadas. Piensan las cosas antes de emitir opinión o tomar decisiones. Son muy serviciales y colaboradoras en las actividades que desarrollan, se adaptan bien al trabajo en grupos y si aceptan un compromiso de trabajo o tarea, se puede confiar en que la van terminar con precisión y exactitud. Son personas que les gusta que reconozcan su trabajo y además las motiva, pero no se dejan halagar si su trabajo no está bien, no aceptan halagos falsos o superficiales.

En el cuadrante PgAR.IV, se ubicaron 19 estudiantes (32\%), son abstractivos/introvertidos, esto propicia que sean estudiantes analíticos y muy meticulosos, por lo que se inclinan a buscar y recabar datos, esto los hace personas auto-disciplinadas, trabajadoras, abnegadas, estéticas y con gran capacidad de análisis crítico. Les gusta la cortesía y son reservadas. Las cosas las hacen muy bien hechas, ser perfeccionistas y detallistas los orienta por la excelencia y calidad, por lo que necesitan tiempo para hacer las cosas. $\mathrm{Su}$ autodisciplina los ayuda a planificar y trabajar en exceso para cumplir con sus compromisos, dejando aun sus propias necesidades descanso y diversiones (Marston, 1928; Jung, 2000; PDA, 2005; PDA, s.f; Bradberry, 2008; Orozco-Edelman et al., 2017).

Cabe reflexionar que la tendencia de la personalidad de los estudiantes evaluados es hacia la abstracción/introversión, la reflexión y el análisis (62\%). Estos resultados hacen plantear las preguntas ¿es necesario incluir estos procesos de personalidad en el currículo de los estudiantes de pedagogía? ¿Son fundamentales para la docencia los procesos de personalidad estudiantil? ¿Generaría mejores aprendizajes los estudiantes al relacionar su personalidad con su formación universitaria? 
RECIE. Revista Electrónica Científica de Investigación Educativa Vol. 4, núm. 2, enero-diciembre 2019, pp. 1073-1081.

\section{Conclusiones}

Los rasgos de personalidad que se establecieron en las 4 dimensiones o cuadrantes, evidencian que hay estudiantes enérgicos, directos y decididos, que son asertivos, concisos y determinantes. Otros en cambio sus rasgos evidencian que son optimistas, amistosos y muy comunicativos, expresivos, emocionales, populares y amigables. Asimismo, estudiantes que les gusta escuchar, trabajar en equipo y muy tranquilos, son personas estables, pacientes, leales y prácticas. Además, hay otros que les encanta reunir datos, les gusta los detalles y son muy meticulosos en todo lo que hacen, son estudiantes precisos, sensibles, diplomáticos y analíticos. Para la educación es muy significativo, que se evidencie información empírica sobre rasgos de personalidad y su incidencia en los procesos educativos.

Aunque las personas poseen y utilizan sus conocimientos sobre personalidad, estos son menos distintivos y muy solapados si no se realizan procesos de auto-análisis y auto-evaluación, que genere auto-conocimiento. Es por eso que la totalidad de estudiantes al analizar sus resultados, manifestaron sorpresa y admiración al ver objetivamente sus rasgos de su personalidad. Los rasgos que presenta la prueba UPEA, son importantes porque los procesos de la personalidad son fundamentes para la enseñanzaaprendizaje, y están presentes durante toda la vida, se consolidan a partir de las interrelaciones y las interdependencias históricas y socio-culturales de la existencia, donde la educación tiene un apartado fundamental.

\section{Referencias}

Bradberry, T. (2008). El código de la personalidad. Colombia: Norma.

Caicedo, H. (2012) Neuroaprendizaje. Bogotá: Ediciones de la U.

Garbarini, M. (2011). Las características personales del docente y su relación con el rendimiento académico y el aprendizaje significativo de sus alumnos. Argentina: Facultad de Desarrollo e Investigación Educativos. Universidad Abierta Interamericana.

González, C. (2001). El proceso enseñanza-aprendizaje en la educación superior: una reflexión en torno a la Universidad de San Carlos de Guatemala. Guatemala: División de Desarrollo Académico, Universidad de San Carlos de Guatemala.

González, C. (2007). Pensamiento pedagógico, (Vol. 1). Guatemala: Pronice. González, F. (2002). Sujeto y Subjetividad. México: Thomson. Jung C. (2000). Tipos Psicológicos. México: Sudamericana.

Marston, W. (1928). Emotions of Normal People. London: Osmania University Library. 
Mitjáns, A. (2014). Subjetividad, Complejidad y Educación. Psicología para América Latina, 13, pp. 1-19.

Morán M., Fínez M. y Fernández, E. (2017) Sobre la felicidad y su relación con tipos y rasgos de personalidad, Clínica y Salud, 28, pp. 59-63.

Orozco-Edelman, E. González-Oliva, A. (Julio-diciembre, 2016). Unidades de la personalidad desde la emoción y la actitud -UPEA-. Revista del Colegio de Psicólogos de Guatemala, 6(19), pp. 57-64.

Orozco-Edelman, E. Moreno, M. Gómez, D. Osorio, E. y García, L. (2017). Personalidad y educación superior. Guatemala: División de Desarrollo Académico, Universidad de San Carlos de Guatemala.

PDA Internacional. (2005). Manual Técnico. Recuperado de http://www.pdainternational.net/es-gt/PDATheory.aspx

PDA internacional. (s.f). W. M. Marston sustento teórico. Recuperado de http://www.pdainternational.net/es-gt/PDATheory.aspx

Restrepo, J. (2015). Correlatos cognitivos y neuropsicológicos de los cinco grandes: una revisión en el área de la neurociencia de la personalidad. Pensando Psicología, 11, pp. 107-128.

Tirapu, J. (2008) ¿Para qué sirve el cerebro? España: Desclée de Brouwer.

Vásquez, S., Difabio, H. y Noriega, M. (2016) Perspectiva temporal y estilos de personalidad en estudiantes Argentinos. Interdisciplinaria, 33 (2), pp. 315-336.

\section{Agradecimientos}

A la Facultad de Humanidades y al Departamento de Investigación Educativa de la DDA-DIGED, de la Universidad de San Carlos de Guatemala, especialmente al personal académico y auxiliares de investigación. 
RECIE. Revista Electrónica Científica de Investigación Educativa Vol. 4, núm. 2, enero-diciembre 2019, pp. 1073-1081. 